\title{
The Expression of VGF is Reduced in Leukocytes of Depressed Patients and it is Restored by Effective Antidepressant Treatment
}

\author{
Annamaria Cattaneo ${ }^{1,2}$, Antonella Sesta ${ }^{1,2}$, Francesca Calabrese ${ }^{3}$, Gabriela Nielsen ${ }^{4}$, Marco Andrea Riva ${ }^{3,5}$ \\ and Massimo Gennarelli*,1,2
}

'Division of Biology and Genetics, Department of Biomedical Sciences and Biotechnology, University of Brescia, Brescia, Italy; ${ }^{2}$ Genetics Unit, IRCCS San Giovanni di Dio, Fatebenefratelli, Brescia, Italy; ${ }^{3}$ Department of Pharmacological Sciences, Center of Neuropharmacology, Università degli studi di Milano, Milan, Italy; ${ }^{4}$ Psychiatric Unit, IRCCS San Giovanni di Dio, Fatebenefratelli, Brescia, Italy; ${ }^{5}$ Center of Excellence on Neurodegenerative Diseases, Università degli studi di Milano, Milan, Italy

\begin{abstract}
Major depression is a disease characterized by an inability of neuronal systems to show appropriate adaptive plasticity especially under challenging conditions, such as stress. Conversely, pharmacological intervention may normalize such defects through the modulation of factors that might act in concert for the functional recovery of depressed patients, like the neuropeptide VGF, which has previously shown to possess antidepressant like activity. We analyzed VGF mRNA levels in the brain of rodents exposed to stress or treated with antidepressant drugs. In addition, we assessed VGF expression in leukocytes obtained from 25 drug-free depressed patients before and during antidepressant treatment. We found a persistent reduction of VGF expression after exposure to prenatal stress and an upregulation of its levels following chronic treatment with different antidepressant drugs. Moreover, VGF mRNA levels were significantly reduced in drug-free depressed patients, as compared with controls, and were modulated in response to effective antidepressant treatment. Our data provide further support to the role of VGF in mood disorders and suggest that VGF could be a more specific biomarker for treatment responsiveness.
\end{abstract}

Neuropsychopharmacology (2010) 35, |423-1428; doi:I0.1038/npp.2010.1 I; published online 17 March 2010

Keywords: VGF; leukocytes; gene expression; major depression; antidepressants; animal models

\section{INTRODUCTION}

Major depression (MD) is a severe psychiatric disorder with complex and multifactorial etiology originating from the interaction between environmental and genetic factors (Uher, 2008). According to recent hypotheses, MD may be characterized by an inability of neuronal systems to show appropriate adaptive plasticity especially under challenging conditions such as stress, a key precipitating factor for depression (Pittenger and Duman, 2008). Preclinical studies have shown that stress causes neuronal atrophy and negatively affects synaptic plasticity, whereas chronic antidepressant treatment may restore such deficits through a series of neuroadaptive mechanisms (Pittenger and Duman, 2008; Calabrese et al, 2009; Castrén et al, 2007; Krishnan and Nestler, 2008). One of the molecules

*Correspondence: Dr M Gennarelli, Division of Biology and Genetics, Department of Biomedical Sciences and Biotechnologies, University of Brescia, Genetics Unit, IRCCS San Giovanni di Dio, Fatebenefratelli, Viale Europa II, Brescia 25 I23, Italy, Tel: + 39030 350 | 453, Fax: + 39 030 350 I592, E-mail: genetica@fatebenefratelli.it

Received 7 October 2009; revised 29 December 2009; accepted 14 January 2010 implicated in MD pathogenesis and potentially involved in the mechanism of antidepressant drugs is the neurotrophin brain-derived-neurotrophic factor (BDNF) (Castrén et al, 2007), which enhances synaptic plasticity and may lead to an amelioration of neuronal connections that are defective in vulnerable individuals. In this regard, a number of studies has shown that BDNF levels are reduced in depressed patients (Dwivedi et al, 2003; Sen et al, 2008) as well as in animal models of depression (Pittenger and Duman, 2008; Groves, 2007) and that antidepressant treatment is able to increase BDNF expression in specific brain regions (Krishnan and Nestler, 2008; Kozisek et al, 2008; Calabrese et al, 2007). However, in addition to BDNF, neuronal remodelling is probably associated with the modulation of a wide array of genes that might act in concert for the functional recovery of depressed subjects. An interesting gene is VGF (nonacronymic), which encodes a secreted neuronal peptide precursor, which is subsequently processed by neuroendocrine-specific prohormone convertases into more than 10 different peptides and becomes localized into the endoplasmic reticulum by a secretory leader sequence and it is released in response to depolarization (Levi et al, 2004). VGF was originally 
identified as an NGF-responsive gene but its expression is also highly induced by BDNF in vitro and in vivo (Alder et al, 2003). Interestingly, VGF gene contains a CREB binding site within its promoter, which is critical for BDNF-induced VGF expression (Bozdagi et al, 2008). Thus CREB could provide a common element for regulating both BDNF and VGF expression. Studies conducted in VGF knock out animals suggested a role for VGF also in energy balance, because it controls hypothalamic and autonomic outflow pathways that regulate peripheral energy expenditure (Hahm et al, 1999). Furthermore, VGF is an activity-dependent gene, because the blockade of retinal activity results in decreased VGF expression, whereas lesions of the CNS result in upregulation of VGF expression (Snyder et al, 1998). In addition, VGF is also regulated by paradigms that reflect increases in activity such as learning, long-term potentiation, seizure and synaptogenesis (Snyder et al, 1998; Bozdagi et al, 2008).

Finally, VGF levels are reduced in animal paradigms of depression (Thakker-Varia et al, 2007), whereas its expression can be upregulated following ECS or chronic imipramine treatment (Newton et al, 2003; Conti et al, 2007; Altar et al, 2004; Thakker-Varia et al, 2007), consistent with the antidepressant activity of VGF (Thakker-Varia et al, 2007; Hunsberger et al, 2007).

In this study, we provide further evidence for a role of VGF in depression and antidepressant response by conducting a series of studies in rodents exposed to stress or treated with antidepressant drugs. In addition, we provide the first evidence that VGF mRNA levels are reduced in leukocytes of drug-free depressed patients, and are modulated in response to effective antidepressant treatment.

\section{MATERIALS AND METHODS}

\section{Animal Studies}

Rats were individually housed in $40 \times 25 \times 20 \mathrm{~cm}$ Plexiglas boxes, with a metal top and sawdust as bedding, and were maintained in an air-conditioned room (temperature $21 \mathrm{~T}$ $1-\mathrm{C}$, relative humidity $60 \mathrm{~T} 10 \%$ ) with lights on from 0800 to 2000 hours and with standard pellet chow and tap water freely available.

For the prenatal stress experiment, animals were male offspring of timed-pregnant Sprague-Dawley rats purchased from a commercial breeder (Charles River, Calco, Italy). The pregnant females from gestational day 14 (E14) until delivery as previously described (Fumagalli et al, 2004 were individually placed in transparent plastic and exposed to restrained stress for $45 \mathrm{~min}$, three times a day $(0900,1200$ and 1700 hours). Control pregnant females were left undisturbed in their home cages. All animals gave birth at the normal time (during gestational day 22). Molecular analyses were performed on adult control- and prenatally stressed rats (postnatal day 80).

For the pharmacological study, adult (2-3 months old) male Sprague-Dawley rats (Charles River, Calco, Italy) weighing 225-250 g were treated (by gavage) with vehicle (saline solution), duloxetine $(10 \mathrm{mg} / \mathrm{kg} /$ day $)$, fluoxetine (10 mg/kg/day) or imipramine $(10 \mathrm{mg} / \mathrm{kg} /$ twice daily) for 21 days and killed by decapitation $24 \mathrm{~h}$ after the last administration. The hippocampus and prefrontal cortex were rapidly dissected, frozen on dry ice and stored at $-80^{\circ} \mathrm{C}$ for molecular analysis.

All animal handling and experimental procedures were performed in accordance with the EC (EEC Council Directive 86/609 1987), the Italian legislation on animal experimentation (Decreto Legislativo 116/92), and the National Institutes of Health Guide for the Care and Use of Laboratory Animals. All efforts were made to minimize animal suffering and to reduce the number of animals used.

\section{Human Blood Samples and VGF mRNA Levels}

Subjects. Twenty-five patients (age: 43.57 \pm 9.13 , BMI: $23.55 \pm 3.14,18$ females 7 males) affected by MD were recruited by the Psychiatry Rehabilitation Unit of the IRCCS Centro S Giovanni di Dio FBF, Brescia, Italy. The inclusion criteria were a diagnosis of MD according to either ICD 10 and DSM IV criteria, age 18-65 years, parents of Caucasoid European Ethnicity. Exclusion criteria were a personal history of bipolar affective disorder, schizophrenia, mood incongruent psychotic symptoms, primary substance abuse or primary organic disease, current or actively seeking pregnancy, current treatment with antidepressants, antipsychotics or mood stabilizers or any regular treatment for a medical condition. Moreover, no patient was on oral contraceptive pill. Upon entering the study, the wash-out period was at least of 2 weeks and subsequently patients were treated with escitalopram over a period of 3 months. During this period all the patients were co-treated with benzodiazepines until the eighth week of escitalopram treatment. The Montgomery-Asberg Depression Scale, MADRS, was administered at baseline (T0) to assess the illness severity and during escitalopram treatment, at T8 (after 8 weeks of treatment) and T12 (after 12 weeks of treatment) to evaluate symptom improvement. The response to treatment was defined as a reduction of $50 \%$ of the MADRS score and according to this definition, we classified patients as responders or non responders in relation to the MADRS score reduction value.

Simultaneously to the clinical evaluation, a blood sample collection was performed.

A control group, consisting of 25 subjects (age: $45.68 \pm 10.24$, BMI: $24.64 \pm 2.44,14$ females and 11 males) with a negative anamnesis for any Axis I disorder (confirmed by the MINI interview), a negative family history for psychoses and mood disorders and without any regular treatment for a medical condition was enrolled in the study. No control participant was on oral contraceptive pill.

Venous blood samples for both patients and controls were collected in the morning after an overnight fast (between 0800 hours and 0900 hours) in PaxGene tubes. After blood samples were withdrawn, PaxGene Tubes were stored at $-80^{\circ} \mathrm{C}$ until their processing.

The study was approved by the local ethics committee and all subjects enrolled gave their informed consent to the participation.

\section{Quantification of VGF mRNA Levels by Real Time PCR}

For animal studies total RNA was isolated from tissue of different brain structures by single-step guanidinium 
isothiocyanate/phenol extraction using PureZol RNA isolation reagent (Bio-Rad Laboratories S.r.l. Italia) in accordance with the manufacturer's instructions and quantified by spectrophotometric analysis.

For human blood samples RNA isolation was performed using the PAXGene Blood RNA Kit (Qiagen S.p.A., Milan, MI, Italy) according to the manufacturer's protocols. The RNA quantity was assessed by evaluation of the A260/280 and A260/230 ratios using a Nanodrop spectrometer (NanoDrop Technologies, Wilmington, DE, USA) and the RNA quality was determined using an Agilent Bioanalyzer (Agilent Technologies Italia S.p.A. Cernusco sul Naviglio, MI, Italy). Two micrograms of total RNA were then used for cDNA synthesis and for subsequent gene expression analysis in Real Time PCR. In rodents VGF mRNA levels have been normalized on GAPDH, $\beta$-actin (VGF forward primer: GCTGAGGACGCAGTGT and VGF reverse primer: AAGAGCTCTCCCTGG; ID probe $\beta$-actin: Rn00667869_m1; ID probe GAPDH: Rn99999916_s1), whereas in human leukocytes on the five housekeeping genes $\mathrm{GAPDH}, \beta$-actin, $\beta 2$-microglobulin (B2M), cytochrome $c$-1 (Cyc1) and ATP synthase, $\mathrm{H}+$ transporting mitochondrial F1 complex, subunit (Atp5b) (ID probe VGF: Hs00705044_s1; ID probe B2m: Hs99999907_m1; ID probe Cyc1: Hs00357717_m1; ID probe Atp5b: Hs00969569_m1; ID probe $\beta$-actin: Hs99999903_m1 and ID probe GAPDH: Hs00266705_g1).

Data analyses has been performed according to the comparative $C_{\mathrm{t}}$ method by the use of Applied Biosystem Real Time software, that automatically determines, using the auto $C_{\mathrm{t}}$ determination feature, the optimal baseline and threshold settings.

\section{Statistical Analysis}

Univariate analysis of variance was used for comparing the mean values of VGF mRNA levels in drug-free patients $v s$ controls, whereas clinical and biological changes during treatment were analyzed by means of the General Linear Model according to a repeated measures design with time (T0, T8 and T12) as a within-subjects factor. The Pearson's coefficient was used to evaluate bivariate correlations. Statistical comparisons in animals were carried out using one-way analysis of variance (SPSS version 13.0 software package, website: http://www.spss.com).

\section{RESULTS}

\section{Modulation of VGF mRNA Levels in Rodents}

We found that VGF mRNA levels are significantly reduced in the hippocampus of prenatally stressed animals $(-27 \%$ $p=0.013$, Figure 1 ), an experimental paradigm that, in line with the developmental hypothesis of mood disorders, results in persistent functional alterations leading to heightened susceptibility for cognitive and neuropsychiatric disorders (Fumagalli et al, 2007). Moreover, in agreement with previous results (Thakker-Varia et al, 2007; Altar et al, 2004) we found that chronic treatment with different classes of antidepressants, imipramine, fluoxetine and duloxetine, can regulate the expression of VGF. Indeed a significant increase in the expression of the peptide was found in hippocampus (imipramine, $+28 \% p=0.0008$; fluoxetine, +

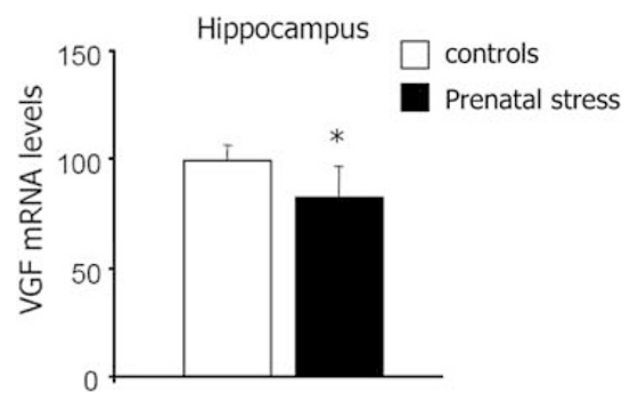

Figure I VGF mRNA levels are reduced following prenatal stress paradigm in rodents. VGF mRNA levels, analyzed by Real Time PCR were significantly reduced in the hippocampus of rats exposed to prenatal stress, as compared with saline-treated rats $(-27 \% p=0.013$, Figure I). The data expressed as mean percentage of the control group (the group that was not exposed to stress or that received vehicle injection), are the mean \pm SEM from 6 to 14 independent determinations. * $p<0.05$ vs control group (One-way ANOVA).

$20 \% p=0.001$; duloxetine, $+20 \% p=0.005$, Figure $2 \mathrm{a})$ as well as in prefrontal cortex (imipramine, $+38 \% p=0.014$; fluoxetine, $+32 \% p=0.036$; duloxetine $+28 \% p=0.04$ ) of chronically treated animals (Figure $2 \mathrm{~b}$ ).

\section{VGF mRNA Levels in Healthy Subjects and Depressed Patients}

We investigated VGF mRNA expression also in human leukocytes to establish a potential relationship between preclinical data and the clinical setting. We found a significant decrease in VGF mRNA levels in drug-free depressed patients (average MADRS scores 21.42 \pm 3.17 ) with respect to their matched controls ( $\mathrm{PT} 0=0.67 \pm 0.46$, $C=1.24 \pm 0.77, \mathrm{~F}=9.02, p=0.004$, Figure $3 \mathrm{a}$ ), although no correlation was found between VGF mRNA levels at T0 and the baseline severity of illness $(\boldsymbol{r}=-\mathbf{0 . 0 9} ; p=0.970)$. No effects of gender, BMI and age on VGF levels were observed in this group of patients $(p=0.438, p=0.343$ and $p=0.512$, respectively). Pharmacological treatment with escitalopram improved the depressive symptomatology at T8 and T12 (mean MADRS scores values of $11.23 \pm 7.07$ and $7.23 \pm 5.15$, respectively) with a significant decrease in the MADRS scores between T8 and T0 $(p<0.001)$ and between T12 and T0 $(p<0.001)$ and with a percentage improvement at T12 of $67.71 \%$. Interestingly, escitalopram treatment significantly modulated VGF expression in depressed subjects $(p<$ $0.001)$. In fact, a planned repeated measure analysis indicated that VGF mRNA levels at T8 were not significantly different from those observed at T0 (PT8 $=0.87 \pm$ $0.75, p=0.099)$, but increased significantly at $\mathrm{T} 12$, (PT12 = 1.52 $\pm 1.07, p<0.001$ vs PT0, Figure 3a). In the same group of controls and patients we have also analyzed the expression levels of the neurotrophin BDNF and, interestingly, we observed a pattern of modulation similar to VGF. In fact, BDNF mRNA levels were significantly reduced in drug-free depressed patients at the baseline (PT0) as compared with controls and were restored to normal levels following 12 weeks of antidepressant treatment (Cattaneo et al, 2010). In addition, to extrapolate some indications on the putative interaction between BDNF and VGF, we calculated a Pearson's correlation factor between the changes of the two trophic molecules induced by 
a

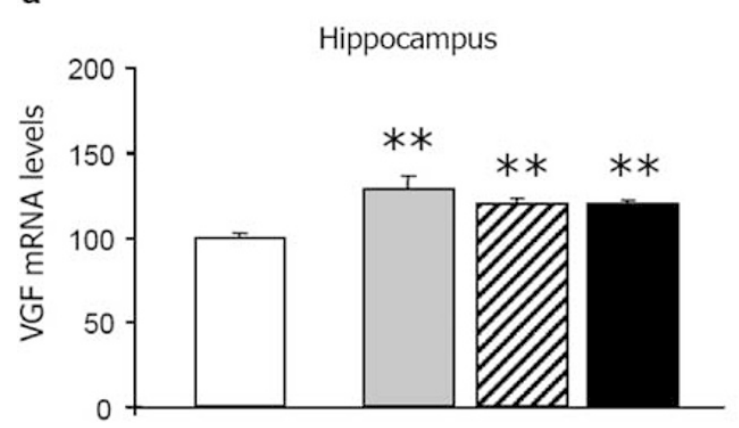

b

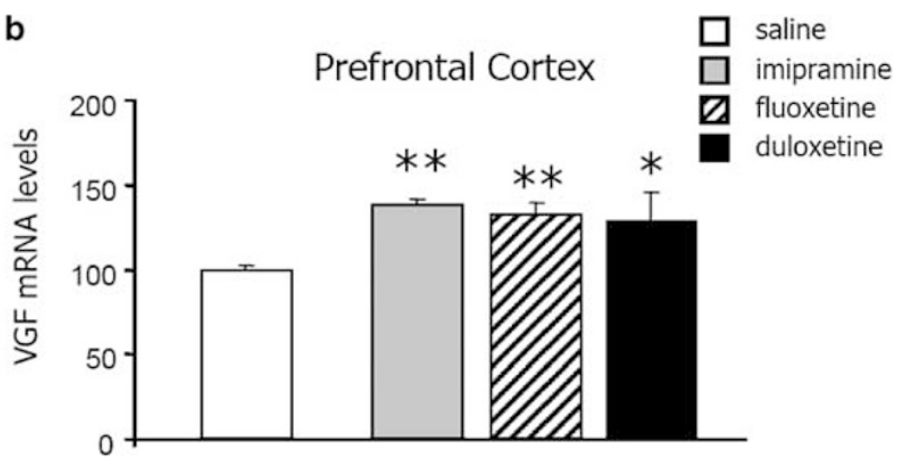

Figure 2 VGF mRNA levels are induced by chronic antidepressant treatment in rodents. VGF mRNA levels were increased following chronic treatment with different antidepressant drugs in rat hippocampus (imipramine, $+28 \% p<0.00$ I; fluoxetine, $+20 \% p=0.00$ l; duloxetine, $+20 \% p=0.005$, (a)) and prefrontal cortex (imipramine, $+38 \% p=0.014$; fluoxetine, $+32 \% p=0.036$; duloxetine $+28 \% p=0.04$, (b)). The data expressed as mean percentage of the control group (the group that was not exposed to stress or that received vehicle injection), are the mean \pm SEM from 6 to 14 independent determinations. ${ }^{*} p<0.05$, $* * p<0.00$ I vs control group (One-way ANOVA).
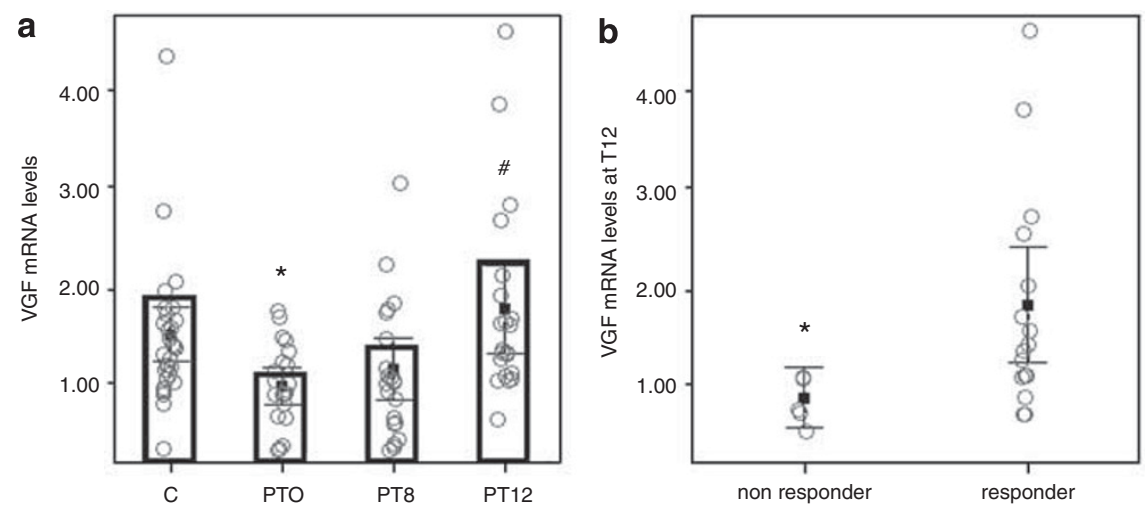

Figure 3 VGF mRNA levels in leukocytes of healthy controls $(C)$ and in depressed patients $(P)$ at three different sample times. VGF mRNA levels were analyzed by Real Time PCR in leukocytes of 25 healthy controls and 25 depressed patients at three different sample times: at baseline (PT0), when patients were drug free for at least 2 weeks, and after 8 (PT8) and 12 (PTI2) weeks of escitalopram treatment. VGF mRNA levels were significantly reduced in drugfree depressed patients as compared with controls (PTO $=0.67 \pm 0.7 \mathrm{I}, C=1.24 \pm 0.77, F=9.02, p=0.004$ ), and escitalopram treatment reversed VGF deficiency (PTI $=1.52 \pm 1.07, p<0.00$ I vs PT0, (a). In particular, after 8 weeks of treatment VGF levels were not different from those observed at the baseline, conversely, 12 weeks of treatment were able to significantly increase VGF levels but only in patients $(n=20)$ whose symptoms ameliorated $(b)$. VGF mRNA levels are expressed in arbitrary units (AU) as a Relative Expression Ratio of the target gene in comparison to the geometric mean of reference genes. ${ }^{*} p<0.05$ PTO vs C, ${ }^{*} p<0.00$ I in PTI 2 vs PTO in a; $* p<0.05$ in responders vs non responders in $b$.

treatment in each patient and, interestingly, we find a positive association $(r=0.577 ; p=0.012)$.

Although changes in VGF levels ( $\triangle \mathrm{VGF}$ mRNA levels) observed in the whole sample during escitalopram treatment did not correlate with symptoms improvement ( $\triangle$ MADRS score, $r=0.276 ; p=0.226$ ), we found that VGF mRNA levels after 12 weeks of escitalopram treatment increased only in patients whose depressive symptoms ameliorated during the course of treatment $(\mathrm{PT} 12=1.76 \pm$ 1.02 ), but not in non responders (PT12 $=0.82 \pm 0.32$, $p=0.040 v s$ treatment responders, Figure $3 \mathrm{~b}$ ). In particular, the percentage increase in VGF levels ( $\triangle$ VGF mRNA levels) after 12 weeks of treatment was of $55.36 \%$ in responder patients and of $24.17 \%$ in non responders $(p=0.006$ responders $v s$ non responders).

\section{DISCUSSION}

The data of our study show that the expression of VGF is reduced in rodents under a condition that may enhance the susceptibility to depression and it is significantly upregulated after chronic antidepressant treatment. Moreover, we show for the first time that similar changes are also found in depressed subjects who show reduced levels of VGF at baseline, which are normalized in patients who respond to pharmacological therapy.

It is generally accepted that antidepressant drugs may exert their therapeutic efficacy by improving synaptic plasticity, through the modulation of a number of molecules, which may enhance neuronal resilience (Pittenger and Duman, 2008). Different peptides with trophic properties, including the neurotrophin BDNF (Pittenger and Duman, 2008; Castrén et al, 2007; Groves, 2007; Kozisek et al, 2008; Calabrese et al, 2007), FGF2 (Turner et al, 2006) and VEGF (Warner-Schmidt and Duman, 2008), have antidepressant activity and their expression is upregulated by pharmacological treatment.

VGF has only recently been proposed as a neuropeptide that may mediate antidepressant responses (Thakker-Varia et al, 2007; Hunsberger et al, 2007). Its expression is reduced in depression paradigms, including learned helplessness and 
forced swim test, whereas exogenous administration of VGF exerts antidepressant activity (Thakker-Varia et al, 2007; Hunsberger et al, 2007). We show that exposure to prenatal stress produces a persistent reduction of VGF in rat hippocampus that in association with decreased expression of other neurotrophic molecules may lead to reduced plasticity and enhanced vulnerability to psychiatric disorders that represent a long-term consequence of exposure to adversities early in life (Fumagalli et al, 2007). We also show that chronic treatment with different antidepressant drugs is able to upregulate VGF expression, suggesting that the neuropeptide might represent a common target for antidepressant action. These data are in agreement with previous reports showing that chronic imipramine or ECT can increase the level of VGF in the rat hippocampus (Thakker-Varia et al, 2007; Altar et al, 2004). We have previously shown that BDNF expression was increased in the same animals chronically treated with duloxetine (Calabrese et al, 2007; Molteni et al, 2009). As BDNF and VGF genes are characterized by the presence of the same responsive sequence for the transcription factor CREB it is feasible that these trophic molecules may be modulated through similar mechanisms and/or that can reciprocally modulate each other.

Interestingly, we report for the first time that VGF may undergo similar changes in peripheral cells from depressed subjects. Although the role of VGF in leukocytes remains to be established, these cells show gene expression profiles similar to neurons, which may reflect what is occurring in the brain (Sullivan et al, 2006). In addition similar changes occurring in the brain of patients with psychosis or mood disorders have been observed also in leukocytes supporting the usefulness of these cells to investigate the neurobiology of psychiatric diseases. We found that VGF mRNA levels are significantly reduced in the leukocytes of drug-free depressed patients, as compared with controls, although there is no correlation with the baseline severity of illness. Interestingly, 12 weeks of treatment with the SSRI drug escitalopram upregulate VGF expression although only in responder patients, suggesting that changes in the expression of the neuropeptide may be important for the mechanisms associated with drug response. The above evidence share many similarities to what is known about the peripheral modulation of BDNF in depressed subjects (Krishnan and Nestler, 2008). In this regard, analyzing BDNF expression levels in the same group of controls and patients we have recently found a decrease of BDNF levels in drug-free depressed patients and a progressive increase following escitalopram treatment (Cattaneo et al, 2010). Interestingly, we found a positive correlation between the changes occurring in the expression of BDNF and VGF after antidepressant treatment suggesting that the two molecules could be involved in the same mechanisms induced by antidepressants and/or that they might co-operate in determining the functional recovery of patients.

Interestingly, whereas symptom improvement was already observed after 8 weeks of treatment, the increase of BDNF or VGF was observed only after 12 weeks of treatment. This suggests that there might be a delay between clinical improvement and 'normalization' of neuroplastic defects. Alternatively, it is worth noticing that all patients were co-treated with benzodiazepines but only up to the eighth week. Hence, treatment with benzodiazepines for a short period does not interfere with clinical antidepressant effects, but might limit or prevent the occurrence of neuroplastic changes, such as the modulation of BDNF and VGF. In accordance with this possibility it has been recently shown that benzodiazepines might interfere with the effects of fluoxetine on adult neurogenesis in rodents (Wu and Castrén, 2009; Gass and Henn, 2009).

Although further studies on a larger cohort of patients will be required to confirm the present results, we suggest that VGF could be a more specific biomarker associated with the treatment responsiveness as its expression is increased only in treatment responders, whereas BDNF levels were enhanced by pharmacological treatment independently from clinical amelioration (Krishnan and Nestler, 2008; Brunoni et al, 2008). Serum and plasma BDNF levels can also be reduced in other diseases (Sen et al, 2008) detracting from its use as a diagnostic marker, despite the fact that such changes may be suggestive of common pathophysiological mechanisms. It will be interesting to establish if the changes of VGF can be disease specific or, alternatively, if the combined analysis of different neuropeptides would prove useful for diagnostic purposes as well as to establish treatment responsiveness. In this regard, it has to be noticed that VGF levels have been found altered also in patients with amyotrophic lateral sclerosis (ALS) and Alzheimer's disease (Selle et al, 2005; Pasinetti et al, 2006; Zhao et al, 2008). Under these conditions it has been proposed that changes in VGF expression may promote neurodegeneration, through NMDA- and AMPA-mediated exitotoxic injury. Neurodegeneration is also a feature of $\mathrm{MD}$, because a reduced hippocampal volume accompanied by neuronal atrophy and cell death has been found in depressed patients and dysfunction of the glutamatergic system has been proposed (Sanacora, 2009). Hence, it is possible that a reduction of VGF may represent a common pathophysiological mechanism shared by several disease processes.

The interesting and intriguing finding of our work is that VGF levels increase following AD treatment suggesting an amelioration of neuroplastic processes and more importantly, we observed an increase of VGF only in patients who responded to antidepressant medication suggesting that the trophic molecule may represent a biomarker of treatment outcome. Therefore, it will be interesting to analyze VGF levels during the treatment also in ALS and Alzheimer disease because it should help to monitor the progression of the illness and it should help to clarify and delineate putative functional and therapeutic implication of VGF.

\section{ACKNOWLEDGEMENTS}

We thank Drs Fabio Fumagalli, Raffaella Molteni and Anna Placentino for contributing to part of this study. This research has been supported by grants from the Ministry of University and Research (PRIN no. 2007STRNHK to MAR) Ministry of Health (Ricerca finalizzata 2007 conv/42 to MG and MAR; Ricerca Corrente 2008 to MG) and a liberal contribution from Eli Lilly to MAR.

\section{DISCLOSURE}

All the authors declare they have no competing financial interests. 


\section{REFERENCES}

Alder J, Thakker-Varia S, Bangasser DA, Kuroiwa M, Plummer MR, Shors TJ et al (2003). Brain-derived neurotrophic factorinduced gene expression reveals novel actions of VGF in hippocampal synaptic plasticity. J Neurosci 23: 10800-10808.

Altar CA, Laeng P, Jurata LW, Brockman JA, Lemire A, Bullard J et al (2004). Electroconvulsive seizures regulate gene expression of distinct neurotrophic signaling pathways. J Neurosci 24: 2667-2677.

Bozdagi O, Rich E, Tronel S, Sadahiro M, Patterson K, Shapiro ML et al (2008). The neurotrophin-inducible gene Vgf regulates hippocampal function and behavior through a brain-derived neurotrophic factordependent mechanism. J Neurosci 28: 9857-9869.

Brunoni AR, Lopes M, Fregni F (2008). A systematic review and meta-analysis of clinical studies on major depression and BDNF levels: implications for the role of neuroplasticity in depression. Int J Neuropsychopharmacol 28: 1-12.

Calabrese F, Molteni R, Maj PF, Cattaneo A, Gennarelli M, Racagni $\mathrm{G}$ et al (2007). Chronic duloxetine treatment induces specific changes in the expression of BDNF transcripts and in the subcellular localization of the neurotrophin protein. Neuropsychopharmacology 32: 2351-2359.

Calabrese F, Molteni R, Racagni G, Riva MA (2009). Neuronal plasticity: a link between stress and mood disorders. Psychoneuroendocrinology 34(Suppl 1): S208-S216.

Castrén E, Võikar V, Rantamäki T (2007). Role of neurotrophic factors in depression. Curr Opin Pharmacol 7: 18-21.

Cattaneo A, Bocchio-Chiavetto L, Zanardini R, Milanesi E, Placentino A, Gennarelli M (2010). Reduced peripheral brainderived neurotrophic factor mRNA levels are normalized by antidepressant treatment. Int J Neuropsychopharmacol 13: 103-108.

Conti B, Maier R, Barr AM, Morale MC, Lu X, Sanna PP et al (2007). Region-specific transcriptional changes following the three antidepressant treatments electro convulsive therapy, sleep deprivation and fluoxetine. Mol Psychiatry 12: 167-189.

Dwivedi Y, Rizavi HS, Conley RR, Roberts RC, Tamminga CA, Pandey GN (2003). Altered gene expression of brain-derived neurotrophic factor and receptor tyrosine kinase $B$ in postmortem brain of suicide subjects. Arch Gen Psychiatry 60: 804-815.

Fumagalli F, Bedogni F, Perez J, Racagni G, Riva MA (2004). Corticostriatal brain-derived neurotrophic factor dysregulation in adult rats following prenatal stress. Eur J Neurosci 20: $1348-1354$

Fumagalli F, Molteni R, Racagni G, Riva MA (2007). Stress during development: Impact on neuroplasticity and relevance to psychopathology. Prog Neurobiol 81: 197-217.

Gass P, Henn FA (2009). Is there a role for neurogenesis in depression? Biol Psychiatry 66: 3-4.

Groves JO (2007). Is it time to reassess the BDNF hypothesis of depression? Mol Psychiatry 12: 1079-1088. Review.

Hahm S, Mizuno TM, Wu TJ, Wisor JP, Priest CA, Kozak CA et al (1999). Targeted deletion of the Vgf gene indicates that the encoded secretory peptide precursor plays a novel role in the regulation of energy balance. Neuron 23: 537-548.

Hunsberger JG, Newton SS, Bennett AH, Duman CH, Russell DS, Salton SR et al (2007). Antidepressant actions of the exerciseregulated gene VGF. Nat Med 13: 1476-1482.

Kozisek ME, Middlemas D, Bylund DB (2008). Brain-derived neurotrophic factor and its receptor tropomyosin-related kinase
B in the mechanism of action of antidepressant therapies. Pharmacol Ther 117: 30-51. Review.

Krishnan V, Nestler EJ (2008). The molecular neurobiology of depression. Nature 455: 894-902.

Levi A, Ferri GL, Watson E, Possenti R, Salton SR (2004). Processing, distribution, and function of VGF, a neuronal and endocrine peptide precursor. Cell Mol Neurobiol 24: 517-533. Review.

Molteni R, Calabrese F, Cattaneo A, Mancini M, Gennarelli M, Racagni $G$ et al (2009). Acute stress responsiveness of the neurotrophin BDNF in the rat hippocampus is modulated by chronic treatment with the antidepressant duloxetine. Neuropsychopharmacology 34: 1523-1532.

Newton SS, Collier EF, Hunsberger J, Adams D, Terwilliger R, Selvanayagam E et al (2003). Gene profile of electroconvulsive seizures: induction of neurotrophic and angiogenic factors. J Neurosci 23: 10841-10851.

Pasinetti GM, Ungar LH, Lange DJ, Yemul S, Deng H, Yuan X et al (2006). Identification of potential Csf biomarkers in Als. Neurology 66: 1218-1222.

Pittenger C, Duman RS (2008). Stress, depression, and neuroplasticity: a convergence of mechanisms. Neuropsychopharmacology 1: 88-109.

Selle H, Lamerz J, Buerger K, Dessauer A, Hager K, Hampel H et al (2005). Identification of novel biomarker candidates by differential peptidomics analysis of cerebrospinal fluid in Alzheimer's disease. Comb Chem High Throughput Screen 8: 801-806.

Sanacora G (2009). Do glutamatergic agents represent a new class of antidepressant drugs? Part 1. J Clin Psychiatry 70: 1473-1475. Review.

Sen S, Duman R, Sanacora G (2008). Serum brain-derived neurotrophic factor, depression, and antidepressant medications: meta-analyses and implications. Biol Psychiatry 64: 527-532.

Snyder SE, Cheng HW, Murray KD, Isackson PJ, McNeill TH, Salton SR (1998). The messenger RNA encoding VGF, a neuronal peptide precursor, is rapidly regulated in the rat central nervous system by neuronal activity, seizure and lesion. Neuroscience $\mathbf{8 2}$ : 7-19.

Sullivan PF, Fan C, Perou CM (2006). Evaluating the comparability of gene expression in blood and brain. Am J Med Genet B Neuropsychiatr Genet 141: 261-268.

Thakker-Varia S, Krol JJ, Nettleton J, Bilimoria PM, Bangasser DA, Shors TJ et al (2007). The neuropeptide VGF produces antidepressant-like behavioral effects and enhances proliferation in the hippocampus. J Neurosci 27: 12156-12167.

Turner CA, Akil H, Watson SJ, Evans SJ (2006). The fibroblast growth factor system and mood disorders. Biol Psychiatry 59: $1128-1135$.

Uher $\mathrm{R}$ (2008). The implications of gene-environment interactions in depression: will cause inform cure? Mol Psychiatry 12: 1070-1078.

Warner-Schmidt JL, Duman RS (2008). VEGF as a potential target for therapeutic intervention in depression. Curr Opin Pharmacol 8: 14-19. Review.

Wu X, Castrén E (2009). Co-treatment with diazepam prevents the effects of fluoxetine on the proliferation and survival of hippocampal dentate granule cells. Biol Psychiatry 66: 5-8.

Zhao Z, Lange DJ, Ho L, Bonini S, Shao Z, Lange DJ et al (2008). Vgf is a novel biomarker associated with muscle weakness in amyotrophic lateral sclerosis (Als), with a potential role in disease pathogenesis. Int J Med Sci 5: 92-99. 